\title{
3-Dimensional Visualization of $\mathrm{na2} / \mathrm{na} 2 \mathrm{Stem}$ in Maize
}

\author{
W. Y. Cheng ${ }^{1}$, D. B. Walden ${ }^{2}$ and P. C. Cheng ${ }^{3}$ \\ ${ }^{1}$ Williamsville East High School, Williamsville, NY 14051 USA \\ ${ }^{2}$ Dept. of Plant Sciences, University of Western Ontario, London, Ontario, N6A 5B7 Canada \\ ${ }^{3}$ Dept. of Electrical Engineering, University at Buffalo, Buffalo, NY 14260 USA
}

The phenotype of nana2 mutant (na2/na2, Fig 6) in maize (Zea mays L.) is characterized by its stubby dwarf appearance. As reported earlier, the longitudinal sections of stem of na2/na2 generally appear similar to the wild-type but with significant by shorter internodes [1] However, in the 2001 summer nursery (an excellent growing season) in London, Ontario, Canada, we observed an interesting characteristic of the mutant in more than 60 homozygous na2/na2 plants. This phenotypic expression was also observed in two plants previously in our 1999 and 1998 summer crop (relatively poor growing sessions).

To visualize the vasculature, mature stems were placed in cellulase-pectinase solution to remove parenchy ma cells. (Cellulase from T. longibrachiatum; Cat. WA18420, and pectinase from A. niger; Cat. 18579; Fluka, Switzerland). This digestion process took 1-6 day s, dep ending on the age of the specimen. Heavily lignified vascular bundles are resistant to the enzymatic digestion and remain intact. To preserve the vascular architecture, the stem segments were pre-glued in a polystyrene frame by cyanoacrylate glue (HobbyTownUSA, Amherst, NY) prior to enzymatic digestion. (This prevents the vascular bundle framework from collap sing after the removal of parenchyma cells.) Serial hand sections (cross section) were digitized by using a modified flat-bed scanner [2], aligned by ImageAcquire software and 3D reconstructed.

The lower portion of the mutant na2/na2 (Fig. 1) stem has a "normal" stem appearance but the internodes length is significantly shortened compared to that found in the wild type (Fig. 1, 2, 3). The cross-sectional views reveal similar nodal (Fig. 5) and internodal (Fig. 4) vascular arrangements, as found in the wild type. However, at a more apical portion of the plant, one frequently observes a continuous "zig-zag" nodal structure; slanted nodes with wedge-shaped internodes characterize this phenotype. Figure 2 shows a longitudinal section cutting through the bi-lateral axis of the stem. Figure 1 shows a longitudinal section with a sagital cut plane, perpendicular to Fig. 2. Sandwiched between the slanted nodes, one finds the initiation of axillary buds (ear: E) apparently supplied by the two joining nodal networks (Fig. 9 macro-stereogram). The leaf-sheath insertion on the plant is also tilted (Fig. 3, indicated by white dotted lines). Figure 7 shows the nodal vasculature in a sagital section. Figure 8 summarize the arrangement of node and internodes in $n a 2 / n a 2$ plant. A diagrammatic representation of the nodal arrangement is shown in Fig.10.

These results suggest that the lower internodes may have been initiated in the embryonic stage assuming the expression of the na2 is not in effect, while the expression of the allele occurs only in the post-germination growth. The tassel internodes in na2/na2 revert to normal, suggesting that this gene expression is "turned off" in the reproductive organs (Fig. 1, 2 and 3).

[1] W. Y. Cheng et al., Microsc. Microanal. 6: Supp 2, (2000) 692-693.

[2] W. Y. Cheng et al., Scanning, 23, (2001)135-136.

[3] W. Y. Cheng et al., Microsc. Microanal., 7: Supp 2, (2001) 444-445.

This article is part of a report by WYC for Siemens Westinghouse Science and Technology Competition (2000, Semi Finalist) and Intel Science Talent Search (2001, Semi-finalist). 


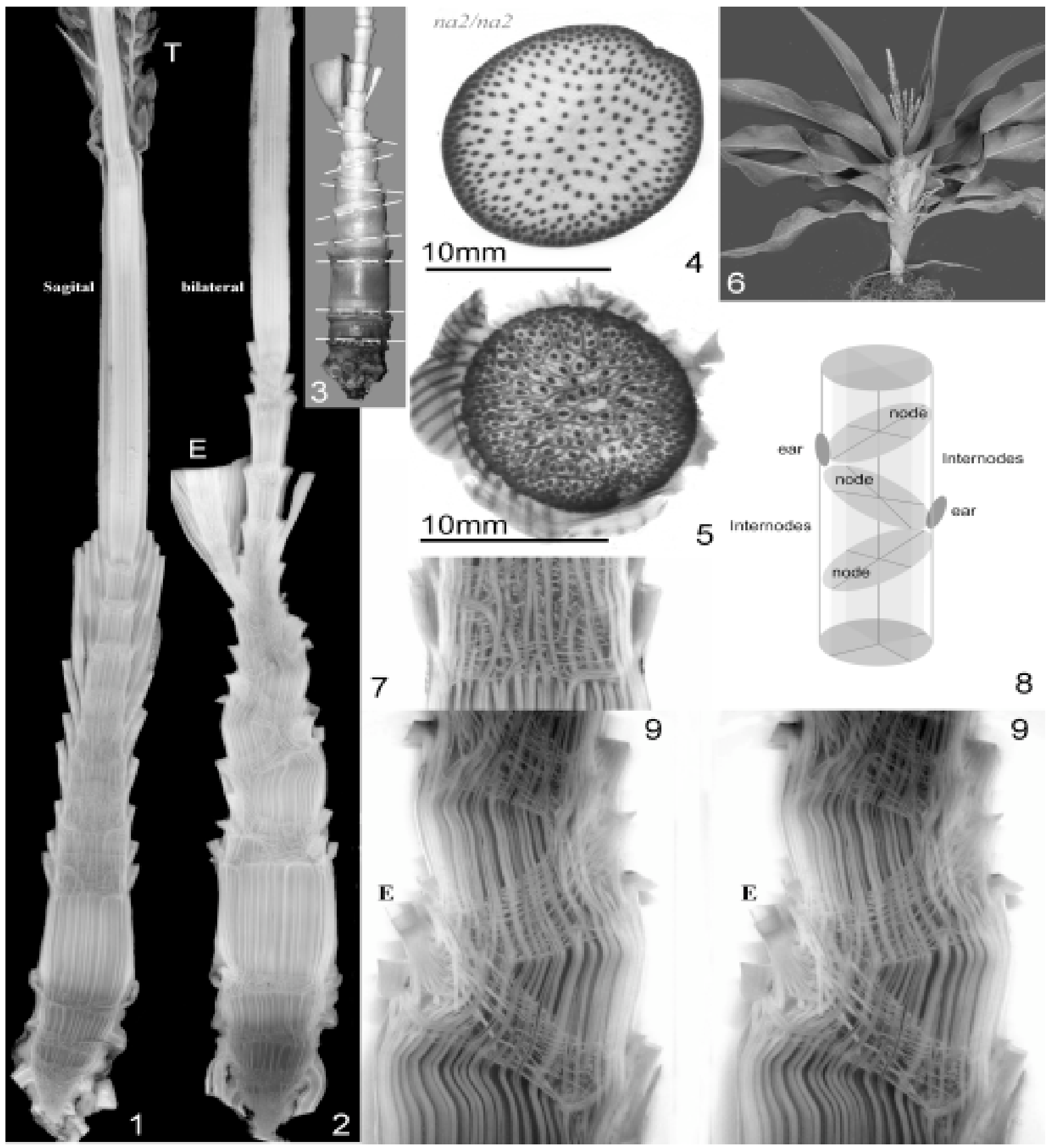

FIG 1 and 2: Sagital and bilateral sections of na2/na2 plants. Note the slanted nodes in the bilateral section. FIG 3: Stem of a na2/na2 plant. Not the slanted leaf sheath insertions.

FIG 4 and 5: Cross sections of na2/na2 stem through internodes and node respectively. FIG 6: Phenotype of na2/na2 plant.

FIG 7: Optical micrograph of the nodal region of na2/na2 plant.

FIG 8: Diagrammatic representation of a $n a 2 / n a 2$ stem showing the slanted nodes and position of ears. FIG 9: Stereogram of a bilateral section of $n a 2 / n a 2$ stem . Note the jointed nodes where ear (E) initiates. 